\title{
Two Methods for Solving Constrained Bi-Matrix Games
}

\author{
Fanyong Meng and Jiaquan Zhan*
}

School of Management, Qingdao Technological University, Qingdao, 266520, China

\begin{abstract}
In real life there are game problems in which players face with certain restrictions in the choice of strategy. These decision problems lead to constrained games. The quadratic programming problem equivalent to a constrained bimatrix game is shown which provides a general method solving constrained bi-matrix games and shows a perfect correspondence between games and programming problems. Besides, a two-step method for constrained games is proposed whose theme is transforming the constrained game into an equivalent ordinary game. In the end, an example is shown to illustrate consistency of the two methods.
\end{abstract}

Keywords: Bi-matrix game, constrained bi-matrix game, quadratic programming.

\section{INTRODUCTION}

There are game problems in real life where the strategies of players are constrained to satisfy certain linear equations or inequalities rather than being in whole strategy space. These decision problems lead to constrained games first introduced by Charnes $[1,2]$. He proved that constrained games can be solved by solving a pair of mutually dual linear programming problems and extended the concept to nperson constrained games. Later it was studied by Kawaguchi and Maruyama [3] in somewhat more generality which widens the scope of game-theoretic approaches. It is shown that the proposed approaches can handle uncertainty in the inequality constraints. More recently, constraints have been introduced in NTU coalitional games and Markov games [4, 5]. Also, a certain constrained dynamic game is shown to be equivalent to a pair of symmetric dual variational problems by Husain and Ahmad [6]. Various duality results are proved under convexity and generalized convexity assumptions on the appropriate functionals. Slightly different from the above-mentioned study, following Charnes's chart, we are more concerned about whether constrained bi-matrix games exist similar results as in constrained matrix games.

\section{PRELIMINARIES ON MATRIX GAMES AND BI- MATRIX GAMES}

In this section, we present certain basic definitions and preliminaries with regard to matrix games and bi-matrix games.

Let $\mathbf{R}^{n}$ denote the $\mathrm{n}$-dimensional Euclidean space and $\mathbf{R}_{+}^{n}$ be its non-negative orthant. Let $A \in R^{m \times n}$ be $\mathrm{m} \times \mathrm{n}$ real matrix and $e^{T}=(1,1, \ldots, 1)$ be a vector of 'ones' whose dimension is specified context. By a two person zero-sum matrix game we mean the triplet $\mathrm{MG}=\left(S^{m}, S^{n}, A\right)$ where
$S^{m}=\left\{x \in \mathbf{R}_{+}^{m}, e^{T} x=1\right\}$ and $S^{n}=\left\{x \in \mathbf{R}_{+}^{n}, e^{T} y=1\right\}$. In the terminology of game theory, $S^{m}$ (respectively $S^{n}$ ) is called the strategy space for Player I (respectively Player II) and $A$ are called the pay-off matrix. Usually two person zero-sum matrix game is abbreviated as matrix game. If Player I chooses $i^{\text {th }}$ pure strategy and Player II chooses $j^{\text {th }}$ pure strategy then $a_{i j}$ is the amount paid by Player II to Player I. The quantity $E(x, y)=x^{T} A y$ is called the expected pay-off of Player I by Player II since elements of $S^{m}$ (respectively $S^{n}$ ) can be thought of as a set of all probability distribution over $I=\{1$, $2, \ldots, m\}$ (respectively $J=\{1,2, \ldots, n\}$ ).

Definition 2.1 (Solution of game). Let $M G=\left(S^{m}, S^{n}, A\right)$ be the given matrix game. A triplet $\left(x^{*}, y^{*}, v^{*}\right) \in S^{m} \times S^{n} \times \mathbf{R}$ is called a solution of the game $M G$ if

$$
E\left(x^{*}, y\right) \geq v^{*}, \forall y \in S^{n},
$$

and

$E\left(x, y^{*}\right) \leq v^{*}, \forall x \in S^{m}$.

Here $x^{*}$ is called an optimal strategy for Player I, $y^{*}$ is called an optimal strategy for PlayerII, $v^{*}$ is called the value of the game $M G$.

Theorem 2.1 (Existence theorem). Let $M G=\left(S^{m}, S^{n}\right.$, $A$ ). Then $\max \min x^{T} A y$ and $\min \max x^{T} A y$ both exists and are equal.

Theorem 2.1 guarantees that every matrix game has a solution. If there is no solution in the pure form then there is certainly a solution in the mixed form. Not long after the invention of simplex method, Kuhn and Tucker et al. [7] pointed out that solving a matrix game is equivalent to solving a pair of primal-dual linear programming.

In matrix game, one player's gain is just the other player's loss. Obviously there are situations in which the interests of two players are not exactly opposite. Such situations give rise to two person non-zero sum matrix games, 
also called bi-matrix games. Some well known bi-matrix game examples are "The Prisoner's Dilemma", "The Battle of Sexes" and "The Bargaining Problem".

A bi-matrix game is expressed as $B G=\left(S^{m}, S^{n}, A, B\right)$, where $A$ and $B$ are $m \times n$ real matrices representing the payoffs to Player I and PlayerII respectively.

Definition 2.2 (Equilibrium point of $\boldsymbol{B} \boldsymbol{G})$. A pair $\left(x^{*}, y^{*}\right)$ $\in S^{m} \times S^{n}$ is said to be an equilibrium point of the bi-matrix game $B G$ if

$$
\begin{aligned}
& \mathrm{x}^{\mathrm{T}} \mathrm{Ay}^{*} \leq \mathrm{x}^{* \mathrm{~T}} \mathrm{Ay}^{*}, \\
& \text { and } \\
& \mathrm{x}^{* \mathrm{~T}} \mathrm{Ay} \leq \mathrm{x}^{* \mathrm{~T}} \mathrm{Ay}^{*}, \\
& \text { for all } x \in S^{m} \text { and } y \in S^{n} .
\end{aligned}
$$

It's straight to find that a matrix game $M G=\left(S^{m}, S^{n}, A\right)$ is a special case of the bi-matrix game $B G$ with $B=-A$.

In the context of bi-matrix game, the following theorem due to Nash is very basic as it guarantees the existence of an equilibrium point of the bi-matrix game $B G$.

Theorem 2.2 (Nash existence theorem [8]). Every bimatrix game $B G=\left(S^{m}, S^{n}, A, B\right)$ has at least one equilibrium point.

As already mentioned that every matrix game can be solved by solving a suitable primal-dual linear programming problems, Mangasarian and Stone [9] established a similar result to show that an equilibrium point of a bi-matrix game can be obtained by solving an appropriate quadratic programming problem.

Theorem 2.3 (Equivalence theorem). Let $B G=\left(S^{m}, S^{n}\right.$, $A, B)$ be the given bi-matrix game. A necessary and sufficient condition that $\left(x^{*}, y^{*}\right)$ be an equilibrium point of $B G$ is that it is a solution of the following quadratic programming problem.

$$
\begin{gathered}
\max x^{T}(A+B) y-\alpha-\beta \\
\text { s.t. }\left\{\begin{array}{c}
A y-\alpha e \leq 0 \\
B^{T} x-\beta e \leq 0 \\
e^{T} x-1=0 \\
e^{T} y-1=0 \\
x, y \geq 0 \\
\alpha, \beta \in \mathbf{R}
\end{array}\right.
\end{gathered}
$$

Further, if $\left(x^{*}, y^{*}, \alpha^{*}, \beta^{*}\right)$ is a solution of the above problem then $\alpha^{*}=x^{* T} A y^{*}, \beta^{*}=x^{* T} B y^{*}$.

If let $B=-A$, theorem 1.3 is reduced to a dual pair of linear programming that is equivalent to a matrix game.

\section{CONSTRAINNED Bİ-MATRIX GAMES AND EQUIVALENT QUADRATIC PROGRAMMING}

There are certain game problems in real life where the strategies of players are constrained to satisfy several linear inequalities rather than being in $S^{m}$ or $S^{n}$ only. These decision problems lead to constrained games.

Let $S_{1}=\left\{x \in \mathbb{R}^{m}, C x \leq c, x \geq 0\right\}, S_{2}=\left\{y \in \mathbb{R}^{n}, D^{T} y \geq d, y \geq\right.$ 0 , where $c \in \mathbb{R}^{s}, d \in \mathbb{R}^{t}, A, B \in R^{m \times n}, C \in R^{s \times m}, D \in R^{n \times t}$. Then the constrained bi-matrix games $C B G$ is denoted as $C B G=$ $\left(S_{1}, S_{2}, A, B\right)$. Note that $e^{T} x=1$ or $e^{T} y=1$ can be rewritten in two inequalities. If $B=-A$, then a constrained bi-matrix game degenerates into a constrained matrix game.

Definition 3.1 (Equilibrium point of $\boldsymbol{C B G})$. A pair $\left(x^{*}\right.$, $\left.y^{*}\right) \in S_{1} \times S_{2}$ is said to be an equilibrium point of the constrained bi-matrix game $C B G$ if

$$
\begin{aligned}
& \mathrm{x}^{\mathrm{T}} \mathrm{Ay}^{*} \leq \mathrm{x}^{* \mathrm{~T}} \mathrm{Ay}^{*}, \\
& \text { and } \\
& \mathrm{x}^{* \mathrm{~T}} \mathrm{By} \leq \mathrm{x}^{* \mathrm{~T}} \mathrm{By}^{*}, \\
& \text { for all } x \in S_{1} \text { and } y \in S_{2} .
\end{aligned}
$$

A main result due to of Charnes [1] in the constrained matrix game theory, as in usual matrix games, assert that every $C M G$ is equivalent to two primal-dual linear programming problems.

Then a natural question is coming: Is there a quadratic programming problem equivalent to a given $C B G$ ? The answer is yes.

Since the constraints are linear, if not empty, the strategy set of Player I, namely $S_{1}$ (respectively $S_{2}$ ) is a convex set on $S^{m}\left(S^{n}\right)$. Let $\left\{x_{1}, x_{2}, \ldots, x_{s}\right\}\left(\left\{y_{1}, y_{2}, \ldots, y_{t}\right\}\right)$ be the vertices of $S_{1}\left(S_{2}\right)$, then we have the following theorem.

Theorem 3.1 (Equivalence theorem of $\boldsymbol{C B G}$ ). Let $C B G$ $=\left(S^{m}, S^{n}, A, B\right)$ be the given constrained bi-matrix game. A necessary and sufficient condition that $\left(x^{*}, y^{*}\right)$ be an equilibrium point of $C B G$ is that it is a solution of the following quadratic programming problem (QPP).

$$
\begin{gathered}
\max x^{T}(A+B) y-\alpha-\beta \\
\text { s.t. }\left\{\begin{array}{c}
x_{i}^{T} A y-\alpha \leq 0 \quad i=1,2, \ldots, s \\
x^{T} B y_{j}-\beta \leq 0 \quad j=1,2, \ldots, t \\
C x \leq c \\
D^{T} y \geq d \\
x, y \geq 0 \\
\alpha, \beta \in \mathbf{R}
\end{array}\right.
\end{gathered}
$$

Further, if $\left(x^{*}, y^{*}, \alpha^{*}, \beta^{*}\right)$ is a solution of the above problem then $\alpha^{*}=x^{* T} A y^{*}, \beta^{*}=x^{* T} B y^{*}$.

Proof. Let $\mathrm{S}$ be the set of all feasible solutions of the above problem. Suppose $\mathrm{S} \neq \varnothing$. For any $(x, y, \alpha, \beta) \in \mathrm{S}$,

$$
x^{T} A y-\alpha=\left(\sum a_{i} x_{i}\right)^{T} A y-\sum a_{i} \alpha=\sum a_{i}\left(x_{i}^{T} A y-\alpha\right) \leq 0 .
$$

Similarly, $x^{T} B y-\beta \leq 0$ and therefore we have $x^{T}(A+B) y$ $-\alpha-\beta \leq 0$. Now suppose that $\left(x^{*}, y^{*}\right)$ is an equilibrium point of the $C B G$. Let $\alpha^{*}=x^{* T} A y^{*}, \beta^{*}=x^{* T} B y^{*}$, then $\left(x^{*}, y^{*}, \alpha^{*}, \beta^{*}\right)$ $\in \mathrm{S}$ and $x^{* T}(A+B) y^{*}-\alpha^{*}-\beta^{*}=0$. Therefore, $\left(x^{*}, y^{*}, \alpha^{*}, \beta^{*}\right)$ is a solution of the above quadratic programming problem and the optimal value of the QPP is 0.

Conversely, let $\left(x^{*}, y^{*}, \alpha^{*}, \beta^{*}\right)$ be a solution of the above QPP, then $\left(x^{*}, y^{*}, \alpha^{*}, \beta^{*}\right) \in \mathrm{S}$ and $x^{* T}(A+B) y^{*}-\alpha^{*}-\beta^{*}=0$. Since $x_{i}^{T} A y^{*}-\alpha^{*} \leq 0$ and $x^{* T} B y_{j}-\beta^{*} \leq 0$, then for arbitrary $(x$, $y) \in S_{1} \times S_{2}$, we have

$$
\mathrm{x}^{\mathrm{T}} \mathrm{Ay}{ }^{*} \leq \alpha^{*} \text { and } \mathrm{x}^{* \mathrm{~T}} \mathrm{By} \leq \beta^{*}
$$


and

$\mathrm{x}^{* \mathrm{~T}} \mathrm{Ay}^{*} \leq \alpha^{*}$ and $\mathrm{x}^{* \mathrm{~T}} \mathrm{By}^{*} \leq \beta^{*}$.

Notice that $x^{* T}(A+B) y^{*}-\alpha^{*}-\beta^{*}=0$, it's straightforward to find that $x^{T} A y^{*} \leq x^{* T} A y^{*}=\alpha^{*}$ and $x^{* T} B y \leq x^{* T} B y^{*}=\beta^{*}$. That is, $\left(x^{*}, y^{*}\right)$ is an equilibrium point of the $C B G$. Here ends the proof.

For a given $C B G$, if there is an equilibrium point of its corresponding $B G$ satisfying the constraints then it certainly is an equilibrium point of the given $C B G$. But even if no equilibrium point of the corresponding $B G$ satisfies the constraints, we can not ensure that the $C B G$ must not have an equilibrium point. Here's a simple example. Consider a bimatrix game with the following payoff matrices

\begin{tabular}{|c|c|}
\hline$(1,1)$ & $(2,0)$ \\
\hline$(0,2)$ & $(1,1)$ \\
\hline
\end{tabular}

The game has only one equilibrium point in mixed form, i.e., $(1 / 2,1 / 2 ; 1 / 2,1 / 2)$. For Player I, assume that the probability of the first strategy being selected must be greater than 0.6. It's not difficult to find the equilibrium point of this constrained bi-matrix game. That is, $(0.6,0.4 ; 1,0)$.

Example 3.1 Consider a bi-matrix game with the following payoff matrices:

$(A, B)=$

\begin{tabular}{|l|l|l|}
\hline$(1,1)$ & $(2,0)$ & $(0,2)$ \\
\hline$(0,2)$ & $(1,1)$ & $(2,0)$ \\
\hline$(2,0)$ & $(0,2)$ & $(1,1)$ \\
\hline
\end{tabular}

Noting that this bi-matrix game is a modified version of the famous rock paper scissors game by adding 'one' to all players' payoff in each situation, then it's straight to find the unique equilibrium point, i.e., $(1 / 3,1 / 3,1 / 3 ; 1 / 3,1 / 3,1 / 3)$. Now suppose that Player I faces with the following restriction:

$x_{1} \geq 0.5$.

Apparently the previous equilibrium point is no longer feasible. Now Player I's strategy space $S_{1}\left\{x \in \mathbb{R}^{m}, x_{1} \geq 0.5\right.$, $\left.e^{T} x=1, x \geq 0\right\}$ is a triangle with three vertices, namely, $\{(1$, $0,0),(1 / 2,1 / 2,0),(1 / 2,0,1 / 2)\}$. Solving the following quadratic programming problem,

$$
\begin{aligned}
\max x^{T}(A+B) y-\alpha-\beta & \\
y_{1}+2 y_{2}-\alpha & \leq 0 \\
y_{1} / 2+3 y_{2} / 2+y_{3}-\alpha & \leq 0 \\
3 y_{1} / 2+y_{2}+y_{3} / 2-\alpha & \leq 0 \\
x_{1}+2 x_{2}-\beta & \leq 0 \\
x_{2}+2 x_{3}-\beta & \leq 0 \\
2 x_{1}+x_{3}-\beta & \leq 0 \\
x_{1}+x_{2}+x_{3} & =1 \\
x_{1} & \geq 0.5 \\
y_{1}+y_{2}+y_{3} & =1 \\
x, y & \geq 0 \\
\alpha, \beta & \in \mathbf{R}
\end{aligned}
$$

we get $x^{*}=(1 / 2,1 / 3,1 / 6)^{T}, y^{*}=(1 / 3,0,2 / 3)^{T}, \alpha^{*}=5 / 6$, $\beta^{*}=7 / 5$

\section{A TWO-STEP METHOD FOR CONSTRAINED GAMES}

Consider a constrained bi-matrix game $C B G$, let $\left\{x_{1}, x_{2}\right.$, $\left.\ldots, x_{s}\right\}\left(\left\{y_{1}, y_{2}, \ldots, y_{t}\right\}\right)$ be the vertices of $S_{1}\left(S_{2}\right)$, then any $x$ $\in S_{1}\left(y \in S_{2}\right)$ can be expressed by a convex combination of $\left\{x_{1}, x_{2}, \ldots, x_{s}\right\}\left(\left\{y_{1}, y_{2}, \ldots, y_{t}\right\}\right.$. Given $(x, y) \in S_{1} \times S_{2}$, Player I's payoff is

$$
\begin{aligned}
& x^{T} A y=\left(\sum_{i} p_{i} x_{i}\right)^{T} A\left(\sum_{j} q_{j} y_{j}\right) \\
& =\left(p_{1}, \ldots, p_{s}\right)\left(\begin{array}{lll}
x_{1} & \ldots & x_{s}
\end{array}\right)_{s \times m}^{T} A_{m \times n}\left(\begin{array}{lll}
y_{1} & \ldots & y_{t}
\end{array}\right)_{n \times t}\left(\begin{array}{c}
q_{1} \\
\vdots \\
q_{t}
\end{array}\right) \\
& =p^{T}\left[\left(\begin{array}{lll}
x_{1} & \ldots & x_{s}
\end{array}\right)_{s \times m}^{T} A_{m \times n}\left(\begin{array}{lll}
y_{1} & \ldots & y_{t}
\end{array}\right)_{n \times t}\right] q \\
& =p^{T} A^{\prime} q \text {, }
\end{aligned}
$$

where $p, q \geq 0$ and $e^{T} p=e^{T} q=1$.

By same operation, Player II's payoff can be rewritten as $p^{T} B^{\prime} q$. So far, the constrained bi-matrix game has been transformed into an ordinary bi-matrix game.

Example 3.1 (continued). In this example, only Player I faces restrictions, hence

$$
\begin{aligned}
A^{\prime} & =\left(\begin{array}{lll}
x_{1} & \ldots & x_{s}
\end{array}\right)_{s \times m}^{T} A_{m \times n} \\
& =\left(\begin{array}{ccc}
1 & 1 / 2 & 1 / 2 \\
0 & 1 / 2 & 0 \\
0 & 0 & 1 / 2
\end{array}\right)^{T}\left(\begin{array}{lll}
1 & 2 & 0 \\
0 & 1 & 2 \\
2 & 0 & 1
\end{array}\right)=\left(\begin{array}{ccc}
1 & 2 & 0 \\
1 / 2 & 3 / 2 & 1 \\
3 / 2 & 1 & 1 / 2
\end{array}\right) \\
B^{\prime} & =\left(\begin{array}{lll}
x_{1} & \ldots & x_{s}
\end{array}\right)_{s \times m}^{T} B_{m \times n}=\left(\begin{array}{ccc}
1 & 0 & 2 \\
3 / 2 & 1 / 2 & 1 \\
1 / 2 & 1 & 3 / 2
\end{array}\right) .
\end{aligned}
$$

According theorem 1.3, solving the quadratic programming problem that is equivalent to the bi-matrix game with payoff matrices $\left(A^{\prime}, B^{\prime}\right)$,

$$
\begin{gathered}
\max p^{T}\left(A^{\prime}+B^{\prime}\right) q-\alpha-\beta \\
\text { s.t. }\left\{\begin{array}{c}
A^{\prime} q-\alpha e \leq 0 \\
B^{\prime T} p-\beta e \leq 0 \\
e^{T} p-1=0 \\
e^{T} q-1=0 \\
p, q \geq 0 \\
\alpha, \beta \in \mathrm{R} /,
\end{array}\right.
\end{gathered}
$$

we get $p^{*}=(0,2 / 3,1 / 3)^{T}, q^{*}=(1 / 3,0,2 / 3)^{T}, \alpha^{*}=5 / 6$, $\beta^{*}=7 / 5$. Not surprisingly, the two methods get the same result since $x^{*}=\sum_{i} p_{i} x_{i}=(1 / 2,1 / 3,1 / 6)^{T}$ and $y^{*}=q^{*}$.

Just as already seen, 'unconstrained' or 'constrained' is a relative distinction, a constrained game is an ordinary game itself. As long as the strategy space and payoff function of a constrained game satisfy appropriate conditions, see Glicks- 
berg [10], then this constrained game exists equilibrium. And therefore we do not discuss this issue further here.

\section{CONCLUSION AND DISCUSSION}

In this paper we proposed two methods to deal with constrained bi-matrix games. One is solving equivalent quadratic programming problems and the other is transforming constrained bi-matrix games into ordinary unconstrained bimatrix games. Unlike constrained matrix games, if the constraints change continuously, we still can not trace equilibrium of constrained bi-matrix games dynamically unless we are able to trace the change of strategy set dynamically. Moreover, constraints in actual game problems are not necessarily linear. All these need further studies in the future.

\section{CONFLICT OF INTEREST}

The authors confirm that this article content has no conflict of interest.

\section{ACKNOWLEDGEMENTS}

This work is supported by the National Natural Science Foundation of China (Nos. 71201089), and the Natural Science Foundation Youth Project of Shandong Province, China (ZR2012GQ005).

\section{REFERENCES}

[1] A. Charnes, "Constrained games and linear programming," Proceedings of the National Academy of Sciences of the United States of America, vol. 39, 1953, pp.639-642.

[2] A. Charnes and S. Sorensen, "Constrained n-person games," International Journal of Game Theory, vol. 3, 1974, pp.141-158.

[3] T. Kawaguchi and Y. Maruyama, "Generalized constrained games in farm planning," American Journal of Agricultural Economics, vol. 54, 1972, pp.591-602.

[4] G. Greco, E. Malizia, L. Palopoli and F. Scarcello, "Nontransferable utility coalitional games via mixed-integer linear constraints," Journal of Artificial Intelligence Research, vol. 38, 2010, pp.633-685.

[5] E. Altman, S. Sarkar and E. Solan, "Constrained Markov games with transition probabilities controlled by a single player," In Proceedings of the 2nd international conference on Performance evaluation methodologies and tools, pp.73-78 ICST. 2007

[6] I. Husain and B. Ahmad, "Constrained dynamic game and symmetric duality for variational problems," Journal of Mathematics and System Science, vol. 2, 2012, pp.171-176.

[7] D. Gale, H. W. Kuhn and A. W. Tucker, "Linear programming and the theory of games," Activity Analysis of Production and Allocation, vol. 13, 1951, pp.317-335.

[8] J. F. Nash, "Equilibrium points in n-person games," Proceedings of the National Academy of Sciences, vol. 36, 1950, pp.48-49.

[9] O. L. Mangasarian and H. Stone, "Two-person nonzero-sum games and quadratic programming," Journal of Mathematical Analysis and Applications, vol. 9, 1964, pp. 348-355.

[10] I. L. Glicksberg, "A further generalization of the Kakutani fixed point theorem, with application to Nash equilibrium points," Proceedings of the American Mathematical Society, vol. 3, 1952, pp.170-171. 